\title{
Response to comment on: Schmidt MI, Duncan BB, Vigo $A$ et al. (2006) Leptin and incident type 2 diabetes: risk or protection? Diabetologia 49:2086-2096
}

\author{
M. I. Schmidt • B. B. Duncan • A. Vigo • J. S. Pankow • \\ D. Couper • C. M. Ballantyne • R. C. Hoogeveen • \\ G. Heiss • for the ARIC Investigators
}

Received: 5 September 2006 / Accepted: 8 September 2006 / Published online: 9 November 2006

(C) Springer-Verlag 2006

To the Editor: We thank Snijder et al. for sharing their data from the Hoorn Study [1] and for their interpretation of the protective associations we reported between leptin and incident diabetes in middle-aged adults from the Atherosclerosis Risk in Communities (ARIC) study [2].

The findings of Snijder et al. differ from those we reported. First, in their study, positive leptin associations in minimally adjusted models were only statistically significant among women. In our study, minimally adjusted associations, aside from being more pronounced, were statistically significant for

\footnotetext{
M. I. Schmidt $\cdot$ B. B. Duncan · A. Vigo

Graduate Studies Program in Epidemiology, School of Medicine,

Federal University of Rio Grande do Sul,

Porto Alegre, RS, Brazil

M. I. Schmidt • B. B. Duncan $\cdot$ G. Heiss

Department of Epidemiology, School of Public Health,

University of North Carolina,

Chapel Hill, NC, USA

J. S. Pankow

Division of Epidemiology, School of Public Health,

University of Minnesota,

Minneapolis, MN, USA

D. Couper

Department of Biostatistics, School of Public Health, University of North Carolina,

Chapel Hill, NC, USA

C. M. Ballantyne $\cdot$ R. C. Hoogeveen

Department of Medicine, Baylor College of Medicine,

Houston, TX, USA

M. I. Schmidt $(\square)$

School of Medicine, UFRGS,

R. Ramiro Barcelos, 2600/414,

Porto Alegre, RS 90035-003, Brazil

e-mail: mischmidt@orion.ufrgs.br
}

both men and women, and were in fact somewhat stronger in men. Second, after multiple adjustments, Snijder et al. found a tendency for a protective association only among men, while we found protective associations among both men and women, with this being somewhat stronger in women. Finally, the Dutch study permitted analysis of diabetes ascertained using both fasting and post-load values, and when these latter cases were considered, the apparent protection decreased in men and disappeared in women.

As the findings of the Hoorn Study are based on relatively few incident cases (51 men and 47 women when the American Diabetes Association 1997 definition was used, and 59 men and 57 women when the WHO 1999 criteria were applied) [1], their study may have lacked power to find statistically significant associations. In contrast, the ARIC study was based on a total of 581 cases (229 men and 352 women) [2]. Small sample size also increases the risk of multicollinearity. Furthermore, the final models of the Hoorn Study appear to be over-parameterised, following the model-building guideline of including at most one independent variable for every ten outcomes [3].

Snijder et al. suggest that our findings may result from ascertainment of diabetes by fasting glucose alone, thus not being true if a wider definition were used. However, $22 \%$ of ARIC cases were ascertained by reported physician diagnosis or medication use. Arguably, cases diagnosed by report are less likely to be misclassified than those based on glucose determination at a single time point; post-load glucose in particular is known to be subject to great variability [4]. Nonetheless, it is possible that leptin's protective associations are more likely to be observed in the fasting state. As obesity is more strongly associated with fasting hyperglycaemia than with post-load hyperglycaemia, leptin, being an adipokine, may play a greater role in fasting than post-load metabolic regulation. 
What else could explain discrepancies between the studies? Chance is always a possibility, particularly as confidence intervals between their findings and ours frequently overlap. Another possibility is that the two study samples differ in important ways. For example, the Hoorn Study had more current smokers and considerably fewer black participants than the ARIC study [1, 2], and protective associations tended to be stronger in black subjects and in non-smokers in ARIC (see Figs 2 and 3 of the original publication) [2]. In addition, the Hoorn Study did not include all the covariates that were used in ARIC, omitting markers of inflammation and quadratic terms.

The main concern raised by Snijder et al. is that by controlling for metabolic variables that may be intermediates in the association between leptin and the development of diabetes, our model may be subject to over-adjustment.

Although this possibility cannot be ruled out, there are several reasons why we consider this unlikely. Leptin, acting as a signal for satiety and stimulating energy consumption, is not thought to cause obesity (or to produce central fat deposition). It has not been found to cause insulin resistance/ hyperinsulinaemia; in fact, in experimental studies, leptin lowers fasting insulin concentrations [5]. Furthermore, it has been shown to stimulate fat oxidation [6], and we know of no studies that suggest it should increase circulating triacylglycerol levels. As leptin activates AMP kinase, its physiological role is to facilitate glucose uptake and, as such, it should not raise fasting glucose concentrations [5]. It has not, to our knowledge, been shown to lower adiponectin levels. Leptin is released by pro-inflammatory mediators and, while heightening the inflammatory response, appears to be more a permissive component of this response than its stimulus [7]. It has, however, been suggested in some studies to increase blood pressure [8], although the change in hazard ratio produced by additional adjustment for hypertension alone is minor, as shown in the Hoorn analyses [1].

Thus, within this conceptual framework we interpret the positive associations found with minimal adjustment as the result of confounding by obesity and several other factors of the metabolic disarray, including leptin resistance, which both raise leptin levels and increase diabetes risk (Fig. 1b). As such, adjustment for all these variables uncovers underlying protective physiological roles of leptin, illustrated in Fig. 1a. In other words, if two individuals have equal values for other metabolic factors, the one with the higher level of fasting leptin would have a smaller risk of developing diabetes than the one with the lower level.

However, as recognised in our paper, epidemiological studies such as ours and Snijder et al. have limited ability to assess independent effects of inter-related variables such as leptin, obesity, inflammation, hyperglycaemia and insulin resistance. In fact, these variables represent parts of a complex web, the origin of which is difficult to determine. a

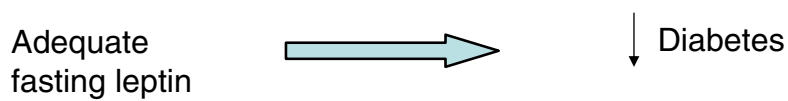

b

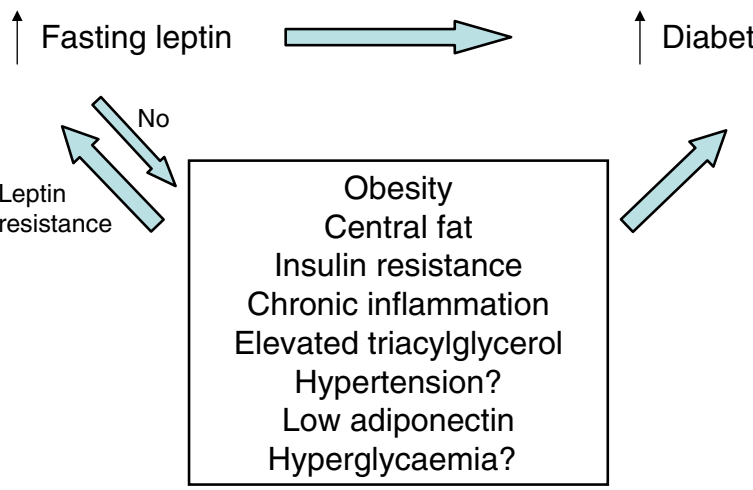

Fig. 1 Analytical framework for the association between fasting leptin levels and incident diabetes in adults. a Main hypothesis: adequate leptin levels protect against diabetes. b Illustration of confounding: diabetes risk factors are not on the pathway between fasting leptin and incident diabetes and are associated with pathways leading to elevated fasting leptin

Thus, we agree with Snijder et al. that caution must be exercised when interpreting associations obtained from observational studies regarding leptin and type 2 diabetes. Nonetheless, in concordance with basic science research, our findings at a population level suggest that models holding other factors constant unveil a basic protective role for leptin in the aetiology of type 2 diabetes.

Duality of interest The authors state that there is no duality of interest.

\section{References}

1. Snijder MB, Dekker JM, Bouter LM, Heine RJ, Stehouwer CD, Seidell JC (2006) Comment on: Schmidt MI, Duncan BB, Vigo A, Pankow JS, Couper D, Ballantyne CM, Hoogeveen RC, Heiss G, for the ARIC Investigators (2006) Leptin and incident type 2 diabetes: risk or protection? Diabetologia 49:2086-2096. Diabetologia DOI 10.1007/s00125-006-0461-7

2. Schmidt MI, Duncan BB, Vigo A et al (2006) Leptin and incident type 2 diabetes: risk or protection? Diabetologia 49:2086-2096

3. Bagley SC, White H, Golomb BA (2001) Logistic regression in the medical literature: standards for use and reporting, with particular attention to one medical domain. J Clin Epidemiol 54:979-985

4. Genuth S, Alberti KG, Bennett P et al (2003) Follow-up report on the diagnosis of diabetes mellitus. Diabetes Care 26:3160-3167

5. Yildiz BO, Haznedaroglu IC (2006) Rethinking leptin and insulin action: therapeutic opportunities for diabetes. Int J Biochem Cell Biol 38:820-830

6. Cohen P, Friedman JM (2004) Leptin and the control of metabolism: role for stearoyl-CoA desaturase-1 (SCD-1). J Nutr 134:2455S-2463S

7. Otero M, Lago R, Lago F et al (2005) Leptin, from fat to inflammation: old questions and new insights. FEBS Lett 579:295-301

8. Haynes WG (2005) Role of leptin in obesity-related hypertension. Exp Physiol 90:683-688 